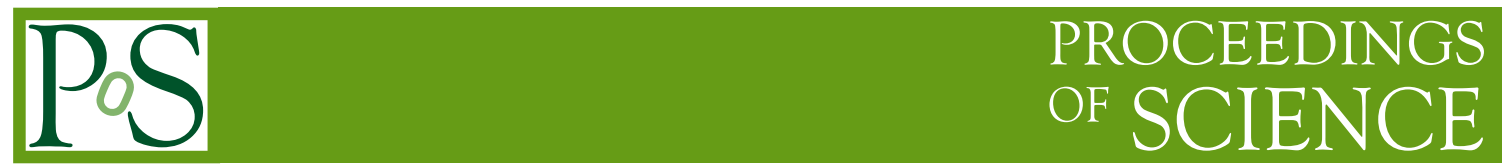

\title{
Phenomenology of Light Sterile Neutrinos
}

\author{
Carlo Giunti* \\ INFN, Sezione di Torino, Via P. Giuria 1, I-10125 Torino, Italy \\ E-mail: giunti@to.infn.it
}

\begin{abstract}
After a short review of the current status of standard three-neutrino mixing, we consider its extension with the addition of one or two light sterile neutrinos which can explain the anomalies found in short-baseline neutrino oscillation experiments. We review the results of the global analyses of short-baseline neutrino oscillation data in $3+1,3+2$ and $3+1+1$ neutrino mixing schemes.
\end{abstract}

Proceedings of the Corfu Summer Institute 2014 "School and Workshops on Elementary Particle Physics and Gravity", 3-21 September 2014, Corfu, Greece

*Speaker. 


\section{Introduction}

Neutrino oscillations have been measured with high accuracy in solar, atmospheric and longbaseline neutrino oscillation experiments. Hence, we know without doubt that neutrinos are massive and mixed particles (see Ref. [1]) and that the Standard Model must be extended in order to take into account these neutrino properties. In this short review we present the status of standard three-neutrino mixing in Section 2 and we discuss the indications in favor of the existence of additional sterile neutrinos given by anomalies found in short-baseline neutrino oscillation experiments in Section 3.

\section{Three-Neutrino Mixing}

Solar neutrino experiments (Homestake [2], GALLEX/GNO [3], SAGE [4], Super-Kamiokande [5], SNO [6], Borexino [7]) measured $v_{e} \rightarrow v_{\mu}, v_{\tau}$ oscillations generated by the solar squaredmass difference $\Delta m_{\text {SOL }}^{2} \simeq 7 \times 10^{-5} \mathrm{eV}^{2}$ and a mixing angle $\sin ^{2} \vartheta_{\text {SOL }} \simeq 0.3$. The KamLAND experiment [8] confirmed these oscillations by observing the disappearance of reactor $\bar{v}_{e}$ with average energy $\langle E\rangle \simeq 4 \mathrm{MeV}$ at the average distance $\langle L\rangle \simeq 180 \mathrm{~km}$.

Atmospheric neutrino experiments (Kamiokande [9], IMB [10], Super-Kamiokande [11], Soudan-2 [12], MACRO [13], MINOS [14]) measured $v_{\mu}$ and $\bar{v}_{\mu}$ disappearance through oscillations generated by the atmospheric squared-mass difference $\Delta m_{\mathrm{ATM}}^{2} \simeq 2.3 \times 10^{-3} \mathrm{eV}^{2}$ and a mixing angle $\sin ^{2} \vartheta_{\text {ATM }} \simeq 0.5$. The K2K [15] and MINOS [16] long-baseline experiments confirmed these oscillations by observing the disappearance of accelerator $v_{\mu}$ with $\langle E\rangle \simeq 1.3 \mathrm{GeV}$ and $3 \mathrm{GeV}$ at distances $L \simeq 250 \mathrm{~km}$ and $730 \mathrm{~km}$, respectively.

The Super-Kamiokande atmospheric neutrino data indicate that the disappearance of $\stackrel{(-)}{v_{\mu}}$ is likely due to $\stackrel{(-)}{v_{\mu}} \rightarrow \stackrel{(-)}{v_{\tau}}$ transitions with a statistical significance of $3.8 \sigma$ [17]. This oscillation channel is confirmed at $4.2 \sigma$ by the observation of four $v_{\mu} \rightarrow v_{\tau}$ events in the OPERA longbaseline accelerator experiment [18] in which the detector was exposed to the CNGS (CERN-Gran Sasso) beam with $\langle E\rangle \simeq 13 \mathrm{GeV}$ at $L \simeq 730 \mathrm{~km}$.

The two independent solar and atmospheric $\Delta m^{2}$ 's are nicely accommodated in the standard framework of three-neutrino mixing in which the left-handed components of the three active flavor neutrino fields $v_{e}, v_{\mu}, v_{\tau}$ are superpositions of three massive neutrino fields $v_{1}, v_{2}, v_{3}$ with masses $m_{1}, m_{2}, m_{3}$ :

$$
v_{\alpha L}=\sum_{k=1}^{3} U_{\alpha k} v_{k L} \quad(\alpha=e, \mu, \tau) .
$$

The unitary mixing matrix can be written in the standard parameterization in terms of three mixing angles $\vartheta_{12}, \vartheta_{23}, \vartheta_{13}$ and a CP-violating phase $^{1} \delta$ :

$$
U=\left(\begin{array}{ccc}
c_{12} c_{13} & s_{12} c_{13} & s_{13} e^{-i \delta} \\
-s_{12} c_{23}-c_{12} s_{23} s_{13} e^{i \delta} & c_{12} c_{23}-s_{12} s_{23} s_{13} e^{i \delta} & s_{23} c_{13} \\
s_{12} s_{23}-c_{12} c_{23} s_{13} e^{i \delta} & -c_{12} s_{23}-s_{12} c_{23} s_{13} e^{i \delta} & c_{23} c_{13}
\end{array}\right)
$$

\footnotetext{
${ }^{1}$ For simplicity, we do not consider the two Majorana CP-violating phases which contribute to neutrino mixing if massive neutrinos are Majorana particles, because they do not affect neutrino oscillations (see Ref. [1]).
} 


\begin{tabular}{lcccccc} 
parameter & $\begin{array}{c}\text { mass } \\
\text { order }\end{array}$ & $\begin{array}{c}\text { best } \\
\text { fit }\end{array}$ & $1 \sigma$ range & $2 \sigma$ range & $3 \sigma$ range & $\begin{array}{c}\text { relative } \\
\text { uncertainty }\end{array}$ \\
\hline$\Delta m_{\mathrm{S}}^{2} / 10^{-5} \mathrm{eV}^{2}$ & & 7.54 & $7.32-7.80$ & $7.15-8.00$ & $6.99-8.18$ & $3 \%$ \\
\hline $\sin ^{2} \vartheta_{12} / 10^{-1}$ & & 3.08 & $2.91-3.25$ & $2.75-3.42$ & $2.59-3.59$ & $5 \%$ \\
\hline \multirow{2}{*}{$\Delta m_{\mathrm{A}}^{2} / 10^{-3} \mathrm{eV}^{2}$} & NO & 2.44 & $2.38-2.52$ & $2.30-2.59$ & $2.22-2.66$ & $3 \%$ \\
& IO & 2.40 & $2.33-2.47$ & $2.25-2.54$ & $2.17-2.61$ & $3 \%$ \\
\hline \multirow{2}{*}{$\sin ^{2} \vartheta_{23} / 10^{-1}$} & NO & 4.25 & $3.98-4.54$ & $3.76-5.06$ & $3.57-6.41$ & $11 \%$ \\
& IO & 4.37 & $4.08-6.10$ & $3.84-6.37$ & $3.63-6.59$ & $11 \%$ \\
\hline \multirow{2}{*}{$\sin ^{2} \vartheta_{13} / 10^{-2}$} & NO & 2.34 & $2.16-2.56$ & $1.97-2.76$ & $1.77-2.97$ & $9 \%$ \\
& IO & 2.39 & $2.18-2.60$ & $1.98-2.80$ & $1.78-3.00$ & $9 \%$ \\
\hline
\end{tabular}

Table 1: Best fit values of the neutrino mixing parameters obtained in the global analysis of neutrino oscillation data presented in Ref. [27] in the framework of three-neutrino mixing with normal ordering (NO) and inverted ordering (IO). The relative uncertainty has been obtained from the $3 \sigma$ range divided by 6 .

where $c_{a b} \equiv \cos \vartheta_{a b}$ and $s_{a b} \equiv \sin \vartheta_{a b}$. It is convenient to choose the numbering of the massive neutrinos in order to have

$$
\Delta m_{\mathrm{SOL}}^{2}=\Delta m_{21}^{2} \ll \Delta m_{\mathrm{ATM}}^{2}=\frac{1}{2}\left|\Delta m_{31}^{2}+\Delta m_{32}^{2}\right|,
$$

with $\Delta m_{k j}^{2}=m_{k}^{2}-m_{j}^{2}$. Then, there are two possible orderings for the neutrino masses: the normal ordering (NO) with $m_{1}<m_{2}<m_{3}$ and the inverted ordering (IO) with $m_{3}<m_{1}<m_{2}$.

With the conventions in Eqs. (2.2) and (2.3), we have $\vartheta_{\text {SOL }}=\vartheta_{12}$ and $\vartheta_{\text {ATM }}=\vartheta_{23}$. Moreover, the mixing angle $\vartheta_{13}$ generates $\stackrel{(-)}{v_{e}}$ disappearance and $\stackrel{(-)}{v_{\mu}} \rightarrow \stackrel{(-)}{v_{e}}$ transitions driven by $\Delta m_{\mathrm{ATM}}^{2}$, which can be observed in long-baseline neutrino oscillation experiments.

In 2011 the T2K experiment reported the first indication of long-baseline $v_{\mu} \rightarrow v_{e}$ transitions [19], followed by the MINOS experiment [20]. More recently, the T2K Collaboration reported a convincing $7.5 \sigma$ observation of $v_{\mu} \rightarrow v_{e}$ transitions through the measurement of $28 v_{e}$ events with an expected background of $4.92 \pm 0.55$ events [21].

The most precise measurement of the value of $\vartheta_{13}$ comes from the measurement of $\bar{v}_{e}$ disappearance in the Daya Bay reactor experiment [22], which has been confirmed by the data of the RENO [23] and Double Chooz [24] reactor experiments:

$$
\sin ^{2} 2 \vartheta_{13}=0.090_{-0.009}^{+0.008} \quad[25] .
$$

Hence, we have a robust evidence of a nonzero value of $\vartheta_{13}$. This is very important, because the measured value of $\vartheta_{13}$ opens promising perspectives for the observation of CP violation in the lepton sector and matter effects in long-baseline oscillation experiments, which could allow to distinguish the normal and inverted neutrino mass orderings (see Ref. [26]).

The three-neutrino mixing parameters have been determined with good precision with global fits of the neutrino oscillation data. In Tab. 1 we report the results of the global fit presented in Ref. [27], which agree, within the uncertainties, with the NuFIT-v1.2 [28] update of the global analysis presented in Ref. [29]. One can see that all the oscillation parameters are determined with precisions between about $3 \%$ and $11 \%$. The largest uncertainty is that of $\vartheta_{23}$, which is known to 
be close to maximal $(\pi / 4)$, but it is not known if it is smaller or larger than $\pi / 4$. For the Dirac CP-violating phase $\delta$, there is an indication in favor of $\delta \approx 3 \pi / 2$, which would give maximal CP violation, but at $3 \sigma$ all the values of $\delta$ are allowed, including the CP-conserving values $\delta=0, \pi$.

\section{Beyond Three-Neutrino Mixing: Sterile Neutrinos}

The completeness of the three-neutrino mixing paradigm has been challenged by the following indications in favor of short-baseline neutrino oscillations, which require the existence of at least one additional squared-mass difference, $\Delta m_{\mathrm{SBL}}^{2}$, which is much larger than $\Delta m_{\mathrm{SOL}}^{2}$ and $\Delta m_{\mathrm{ATM}}^{2}$ :

1. The reactor antineutrino anomaly [30], which is a deficit of the rate of $\bar{v}_{e}$ observed in several short-baseline reactor neutrino experiments in comparison with that expected from a new calculation of the reactor neutrino fluxes $[31,32]$. The statistical significance is about $2.8 \sigma$.

2. The Gallium neutrino anomaly [33-37], consisting in a short-baseline disappearance of $v_{e}$ measured in the Gallium radioactive source experiments GALLEX [38] and SAGE [39] with a statistical significance of about $2.9 \sigma$.

3. The LSND experiment, in which a signal of short-baseline $\bar{v}_{\mu} \rightarrow \bar{v}_{e}$ oscillations has been observed with a statistical significance of about $3.8 \sigma[40,41]$.

In this review, we consider 3+1 [42-45], 3+2 [46-49] and 3+1+1 [50-53] neutrino mixing schemes in which there are one or two additional massive neutrinos at the $\mathrm{eV}$ scale and the masses of the three standard massive neutrinos are much smaller. Since from the LEP measurement of the invisible width of the $Z$ boson we know that there are only three active neutrinos (see Ref. [1]), in the flavor basis the additional massive neutrinos correspond to sterile neutrinos [54], which do not have standard weak interactions.

The possible existence of sterile neutrinos is very interesting, because they are new particles which could give us precious information on the physics beyond the Standard Model (see Ref. [55, 56]). The existence of light sterile neutrinos is also very important for astrophysics (see Ref. [57]) and cosmology (see Ref. [58-61]).

In the $3+1$ scheme, the effective probability of $\stackrel{(-)}{v_{\alpha}} \rightarrow \stackrel{(-)}{v_{\beta}}$ transitions in short-baseline experiments has the two-neutrino-like form [43]

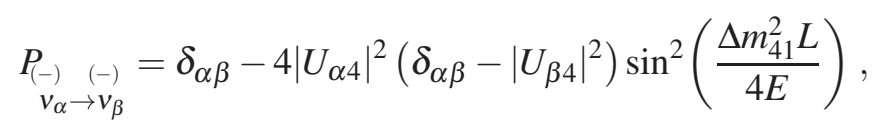

where $U$ is the mixing matrix, $L$ is the source-detector distance, $E$ is the neutrino energy and $\Delta m_{41}^{2}=m_{4}^{2}-m_{1}^{2}=\Delta m_{\mathrm{SBL}}^{2} \sim 1 \mathrm{eV}^{2}$. The electron and muon neutrino and antineutrino appearance and disappearance in short-baseline experiments depend on $\left|U_{e 4}\right|^{2}$ and $\left|U_{\mu 4}\right|^{2}$, which determine the amplitude $\sin ^{2} 2 \vartheta_{e \mu}=4\left|U_{e 4}\right|^{2}\left|U_{\mu 4}\right|^{2}$ of $v_{\mu}^{(-)} \rightarrow \stackrel{(-)}{v_{e}}$ transitions, the amplitude $\sin ^{2} 2 v_{e e}=$ $4\left|U_{e 4}\right|^{2}\left(1-\left|U_{e 4}\right|^{2}\right)$ of $\stackrel{(-)}{v}_{e}$ disappearance, and the amplitude $\sin ^{2} 2 \vartheta_{\mu \mu}=4\left|U_{\mu 4}\right|^{2}\left(1-\left|U_{\mu 4}\right|^{2}\right)$ of ${ }^{(-)} v_{\mu}$ disappearance. 
Since the oscillation probabilities of neutrinos and antineutrinos are related by a complex conjugation of the elements of the mixing matrix (see Ref. [1]), the effective probabilities of shortbaseline $v_{\mu} \rightarrow v_{e}$ and $\bar{v}_{\mu} \rightarrow \bar{v}_{e}$ transitions are equal. Hence, the $3+1$ scheme cannot explain a possible CP-violating difference of $v_{\mu} \rightarrow v_{e}$ and $\bar{v}_{\mu} \rightarrow \bar{v}_{e}$ transitions in short-baseline experiments. In order to allow this possibility, one must consider a $3+2$ scheme, in which, there are four additional effective mixing parameters in short-baseline experiments: $\Delta m_{51}^{2} \geq \Delta m_{41}^{2},\left|U_{e 5}\right|^{2},\left|U_{\mu 5}\right|^{2}$ and $\eta=\arg \left[U_{e 4}^{*} U_{\mu 4} U_{e 5} U_{\mu 5}^{*}\right]$ (see Refs. [62,63]). Since this complex phase appears with different signs in the effective $3+2$ probabilities of short-baseline $v_{\mu} \rightarrow v_{e}$ and $\bar{v}_{\mu} \rightarrow \bar{v}_{e}$ transitions, it can generate measurable $\mathrm{CP}$ violations.

A puzzling feature of the $3+2$ scheme is that it needs the existence of two sterile neutrinos with masses at the $\mathrm{eV}$ scale. We think that it may be considered as more plausible that sterile neutrinos have a hierarchy of masses. Hence, it is interesting to consider also the $3+1+1$ scheme [50-53], in which $m_{5}$ is much heavier than $1 \mathrm{eV}$ and the oscillations due to $\Delta m_{51}^{2}$ are averaged. Hence, in the analysis of short-baseline data in the $3+1+1$ scheme there is one effective parameter less than in the $3+2$ scheme $\left(\Delta m_{51}^{2}\right)$, but CP violations generated by $\eta$ are observable.

Updated global fits of short-baseline neutrino oscillation data have been presented in Refs. [64, 65]. These analyses take into account the final results of the MiniBooNE experiment, which was made in order to check the LSND signal with about one order of magnitude larger distance $(L)$ and energy $(E)$, but the same order of magnitude for the ratio $L / E$ from which neutrino oscillations depend. Unfortunately, the results of the MiniBooNE experiment are ambiguous, because the LSND signal was not seen in neutrino mode $\left(v_{\mu} \rightarrow v_{e}\right)$ [66] and the $\bar{v}_{\mu} \rightarrow \bar{v}_{e}$ signal observed in 2010 [67] with the first half of the antineutrino data was not observed in the second half of the antineutrino data [68]. Moreover, the MiniBooNE data in both neutrino and antineutrino modes show an excess in the low-energy bins which is widely considered to be anomalous because it is at odds with neutrino oscillations $[69,70]^{2}$.

In the following we summarize the results of the analysis of short-baseline data presented in Ref. [65] of the following three groups of experiments:

(A) The $\stackrel{(-)}{v_{\mu}} \rightarrow \stackrel{(-)}{v_{e}}$ appearance data of the LSND [41], MiniBooNE [68], BNL-E776 [73], KARMEN [74], NOMAD [75], ICARUS [76] and OPERA [77] experiments.

(B) The $v_{e}$ disappearance data described in Ref. [37], which take into account the reactor [30-32] and Gallium [33-36,78] anomalies.

(C) The constraints on $\stackrel{(-)}{v}_{\mu}$ disappearance obtained from the data of the CDHSW experiment [79], from the analysis [48] of the data of atmospheric neutrino oscillation experiments ${ }^{3}$, from the analysis [69] of the MINOS neutral-current data [82] and from the analysis of the SciBooNEMiniBooNE neutrino [83] and antineutrino [84] data.

\footnotetext{
${ }^{2}$ The interesting possibility of reconciling the low-energy anomalous data with neutrino oscillations through energy reconstruction effects proposed in Ref. [71,72] still needs a detailed study.

${ }^{3}$ The IceCube data, which could give a marginal contribution [80,81], have not been considered because the analysis is too complicated and subject to large uncertainties.
} 


\begin{tabular}{c|cccc|cc|cc} 
& $3+1$ & $3+1$ & $3+1$ & $3+1$ & $3+2$ & $3+2$ & $3+1+1$ & $3+1+1$ \\
& LOW & HIG & noMB & noLSND & LOW & HIG & LOW & HIG \\
\hline$\chi_{\min }^{2}$ & 291.7 & 261.8 & 236.1 & 278.4 & 284.4 & 256.4 & 289.8 & 259.0 \\
$\mathrm{NDF}$ & 256 & 250 & 218 & 252 & 252 & 246 & 253 & 247 \\
$\mathrm{GoF}$ & $6 \%$ & $29 \%$ & $19 \%$ & $12 \%$ & $8 \%$ & $31 \%$ & $6 \%$ & $29 \%$ \\
\hline$\left(\chi_{\min }^{2}\right)_{\mathrm{APP}}$ & 99.3 & 77.0 & 50.9 & 91.8 & 87.7 & 69.8 & 94.8 & 75.5 \\
$\left(\chi_{\min }^{2}\right)_{\mathrm{DIS}}$ & 180.1 & 180.1 & 180.1 & 180.1 & 179.1 & 179.1 & 180.1 & 180.1 \\
$\Delta \chi_{\mathrm{PG}}^{2}$ & 12.7 & 4.8 & 5.1 & 6.4 & 17.7 & 7.5 & 14.9 & 3.4 \\
$\mathrm{NDF}_{\mathrm{PG}}$ & 2 & 2 & 2 & 2 & 4 & 4 & 3 & 3 \\
$\mathrm{GoF}_{\mathrm{PG}}$ & $0.2 \%$ & $9 \%$ & $8 \%$ & $4 \%$ & $0.1 \%$ & $11 \%$ & $0.2 \%$ & $34 \%$ \\
\hline$\Delta \chi_{\mathrm{NO}}^{2}$ & 47.5 & 46.2 & 47.1 & 8.3 & 54.8 & 51.6 & 49.4 & 49.1 \\
$\mathrm{NDF}$ & 3 & 3 & 3 & 3 & 7 & 7 & 6 & 6 \\
$n \sigma_{\mathrm{NO}}$ & $6.3 \sigma$ & $6.2 \sigma$ & $6.3 \sigma$ & $2.1 \sigma$ & $6.0 \sigma$ & $5.8 \sigma$ & $5.8 \sigma$ & $5.8 \sigma$
\end{tabular}

Table 2: Results of the fit of short-baseline data [65] taking into account all MiniBooNE data (LOW), only the MiniBooNE data above $475 \mathrm{MeV}$ (HIG), without MiniBooNE data (noMB) and without LSND data (noLSND) in the $3+1,3+2$ and $3+1+1$ schemes. The first three lines give the minimum $\chi^{2}\left(\chi_{\mathrm{min}}^{2}\right)$, the number of degrees of freedom (NDF) and the goodness-of-fit (GoF). The following five lines give the quantities relevant for the appearance-disappearance (APP-DIS) parameter goodness-of-fit (PG) [85]. The last three lines give the difference between the $\chi^{2}$ without shortbaseline oscillations and $\chi_{\min }^{2}\left(\Delta \chi_{\mathrm{NO}}^{2}\right)$, the corresponding difference of number of degrees of freedom $\left(\mathrm{NDF}_{\mathrm{NO}}\right)$ and the resulting number of $\sigma$ 's $\left(n \sigma_{\mathrm{NO}}\right)$ for which the absence of oscillations is disfavored.

Table 2 summarizes the statistical results obtained in Ref. [65] from global fits of the data above in the $3+1,3+2$ and $3+1+1$ schemes. In the LOW fits all the MiniBooNE data are considered, including the anomalous low-energy bins, which are omitted in the HIG fits. There is also a 3+1noMB fit without MiniBooNE data and a 3+1-noLSND fit without LSND data.

From Tab. 2, one can see that in all fits which include the LSND data the absence of shortbaseline oscillations is disfavored by about $6 \sigma$, because the improvement of the $\chi^{2}$ with shortbaseline oscillations is much larger than the number of oscillation parameters.

In all the $3+1,3+2$ and $3+1+1$ schemes the goodness-of-fit in the LOW analysis is significantly worse than that in the HIG analysis and the appearance-disappearance parameter goodness-of-fit is much worse. This result confirms the fact that the MiniBooNE low-energy anomaly is incompatible with neutrino oscillations, because it would require a small value of $\Delta m_{41}^{2}$ and a large value of $\sin ^{2} 2 \vartheta_{e \mu}[69,70]$, which are excluded by the data of other experiments (see Ref. [65] for further details $)^{4}$. Note that the appearance-disappearance tension in the $3+2$-LOW fit is even worse than that in the $3+1-\mathrm{LOW}$ fit, since the $\Delta \chi_{\mathrm{PG}}^{2}$ is so much larger that it cannot be compensated by the additional degrees of freedom (this behavior has been explained in Ref. [86]). Therefore, we think that it is very likely that the MiniBooNE low-energy anomaly has an explanation which is different from neutrino oscillations and the HIG fits are more reliable than the LOW fits.

The $3+2$ mixing scheme was considered to be interesting in 2010 when the MiniBooNE neu-

\footnotetext{
${ }^{4}$ One could fit the three anomalous MiniBooNE low-energy bins in a 3+2 scheme [63] by considering the appearance data without the ICARUS [76] and OPERA [77] constraints, but the required large transition probability is excluded by the disappearance data.
} 

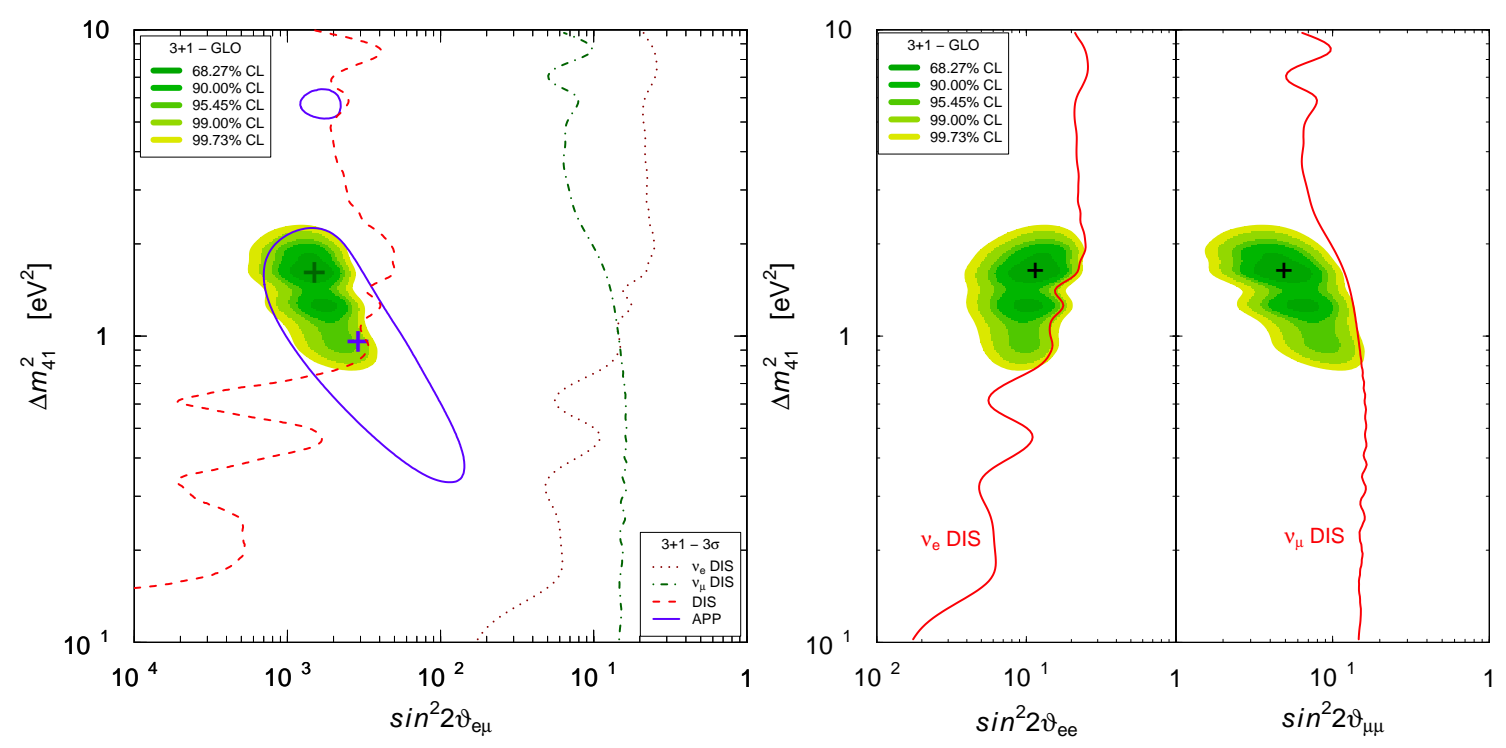

Figure 1: Allowed regions in the $\sin ^{2} 2 \vartheta_{e \mu}-\Delta m_{41}^{2}, \sin ^{2} 2 \vartheta_{e e}-\Delta m_{41}^{2}$ and $\sin ^{2} 2 \vartheta_{\mu \mu}-\Delta m_{41}^{2}$ planes obtained in the global (GLO) 3+1-HIG fit [65] of short-baseline neutrino oscillation data compared with the $3 \sigma$ allowed regions obtained from $\stackrel{(-)}{v_{\mu}} \rightarrow \stackrel{(-)}{v_{e}}$ short-baseline appearance data (APP) and the $3 \sigma$ constraints obtained from $\stackrel{(-)}{v}_{e}$ short-baseline disappearance data $\left(v_{e}\right.$ DIS $), \stackrel{(-)}{v_{\mu}}$ short-baseline disappearance data $\left(v_{\mu}\right.$ DIS $)$ and the combined short-baseline disappearance data (DIS). The best-fit points of the GLO and APP fits are indicated by crosses.

trino [66] and antineutrino [67] data showed a CP-violating tension, but this tension almost disappeared in the final MiniBooNE data [68]. In fact, from Tab. 2 one can see that there is little improvement of the $3+2-\mathrm{HIG}$ fit with respect to the $3+1-\mathrm{HIG}$ fit, in spite of the four additional parameters and the additional possibility of $\mathrm{CP}$ violation. Moreover, since the p-value obtained by restricting the $3+2$ scheme to $3+1$ disfavors the $3+1$ scheme only at $1.2 \sigma$ [65], we think that considering the larger complexity of the $3+2$ scheme is not justified by the data 5 .

The results of the $3+1+1-\mathrm{HIG}$ fit presented in Tab. 2 show that the appearance-disappearance parameter goodness-of-fit is remarkably good, with a $\Delta \chi_{\mathrm{PG}}^{2}$ that is smaller than those in the $3+1$ HIG and $3+2-\mathrm{HIG}$ fits. However, the $\chi_{\min }^{2}$ in the $3+1+1-\mathrm{HIG}$ is only slightly smaller than that in the $3+1-$ HIG fit and the p-value obtained by restricting the $3+1+1$ scheme to $3+1$ disfavors the $3+1$ scheme only at $0.8 \sigma$ [65]. Therefore, there is no compelling reason to prefer the more complex $3+1+1$ to the simpler $3+1$ scheme.

Figure 1 shows the allowed regions in the $\sin ^{2} 2 \vartheta_{e \mu}-\Delta m_{41}^{2}, \sin ^{2} 2 \vartheta_{e e}-\Delta m_{41}^{2}$ and $\sin ^{2} 2 \vartheta_{\mu \mu}-$ $\Delta m_{41}^{2}$ planes obtained in the 3+1-HIG fit of Ref. [65]. These regions are relevant, respectively, for $\stackrel{(-)}{v_{\mu}} \rightarrow \stackrel{(-)}{v_{e}}$ appearance, $\stackrel{(-)}{v_{e}}$ disappearance and $\stackrel{(-)}{v_{\mu}}$ disappearance searches. The corresponding marginal allowed intervals of the oscillation parameters are given in Tab. 3. Figure 1 shows also the region allowed by $\stackrel{(-)}{v_{\mu}} \rightarrow \stackrel{(-)}{v_{e}}$ appearance data and the constraints from $\stackrel{(-)}{v_{e}}$ disappearance and $\stackrel{(-)}{v_{\mu}}$ disappearance data. One can see that the combined disappearance constraint in the $\sin ^{2} 2 \vartheta_{e \mu}-$ $\Delta m_{41}^{2}$ plane excludes a large part of the region allowed by $\stackrel{(-)}{v_{\mu}} \rightarrow \stackrel{(-)}{v_{e}}$ appearance data, leading to the well-known appearance-disappearance tension $[63,64,69,70,86-89]$ quantified by the parameter

\footnotetext{
${ }^{5}$ See however the somewhat different conclusions reached in Ref. [64].
} 


\begin{tabular}{c|cccc}
$\mathrm{CL}$ & $\Delta m_{41}^{2}\left[\mathrm{eV}^{2}\right]$ & $\sin ^{2} 2 \vartheta_{e \mu}$ & $\sin ^{2} 2 \vartheta_{e e}$ & $\sin ^{2} 2 \vartheta_{\mu \mu}$ \\
\hline $68.27 \%$ & $1.55-1.72$ & $0.0012-0.0018$ & $0.089-0.15$ & $0.036-0.065$ \\
\hline $90.00 \%$ & $1.19-1.91$ & $0.001-0.0022$ & $0.072-0.17$ & $0.03-0.085$ \\
\hline $95.00 \%$ & $1.15-1.97$ & $0.00093-0.0023$ & $0.066-0.18$ & $0.028-0.095$ \\
\hline $95.45 \%$ & $1.14-1.97$ & $0.00091-0.0024$ & $0.065-0.18$ & $0.027-0.095$ \\
\hline $99.00 \%$ & $0.87-2.09$ & $0.00078-0.003$ & $0.054-0.2$ & $0.022-0.12$ \\
\hline $99.73 \%$ & $0.82-2.19$ & $0.00066-0.0034$ & $0.047-0.22$ & $0.019-0.14$
\end{tabular}

Table 3: Marginal allowed intervals of the oscillation parameters obtained in the global 3+1-HIG fit of short-baseline neutrino oscillation data [65].

goodness-of-fit in Tab. 2.

It is interesting to investigate what is the impact of the MiniBooNE experiment on the global analysis of short-baseline neutrino oscillation data. With this aim, the authors of Ref. [65] performed two additional 3+1 fits: a 3+1-noMB fit without MiniBooNE data and a 3+1-noLSND fit without LSND data. From Tab. 2 one can see that the results of the 3+1-noMB fit are similar to those of the 3+1-HIG fit and the exclusion of the case of no-oscillations remains at the level of $6 \sigma$. On the other hand, in the 3+1-noLSND fit, without LSND data, the exclusion of the case of no-oscillations drops dramatically to $2.1 \sigma$. In fact, in this case the main indication in favor of short-baseline oscillations is given by the reactor and Gallium anomalies which have a similar statistical significance [37]. Therefore, it is clear that the LSND experiment is still crucial for the indication in favor of short-baseline $\bar{v}_{\mu} \rightarrow \bar{v}_{e}$ transitions and the MiniBooNE experiment has been rather inconclusive.

In conclusion, the results of the global fit of short-baseline neutrino oscillation data presented in Ref. [65] show that the data can be explained by $3+1$ neutrino mixing and this simplest scheme beyond three-neutrino mixing cannot be rejected in favor of the more complex $3+2$ and $3+1+1$ schemes. The low-energy MiniBooNE anomaly cannot be explained by neutrino oscillations in any of these schemes. Moreover, the crucial indication in favor of short-baseline $\bar{v}_{\mu} \rightarrow \bar{v}_{e}$ appearance is still given by the old LSND data and the MiniBooNE experiment has been inconclusive. Hence new experiments are needed in order to check this signal [90-97].

\section{References}

[1] C. Giunti and C.W. Kim, Fundamentals of Neutrino Physics and Astrophysics (Oxford University Press, Oxford, UK, 2007).

[2] Homestake, B.T. Cleveland et al., Astrophys. J. 496 (1998) 505.

[3] GNO, M. Altmann et al., Phys. Lett. B616 (2005) 174, hep-ex/0504037.

[4] SAGE, J.N. Abdurashitov et al., J. Exp. Theor. Phys. 95 (2002) 181, astro-ph/0204245.

[5] Super-Kamiokande, K. Abe et al., Phys. Rev. D83 (2011) 052010, arXiv:1010.0118.

[6] SNO, B. Aharmim et al., Phys.Rev. C88 (2013) 025501, arXiv:1109.0763.

[7] Borexino, G. Bellini et al., Phys.Rev. D89 (2014) 112007, arXiv:1308.0443. 
[8] KamLAND, A. Gando et al., Phys.Rev. D88 (2013) 033001, arXiv:1303.4667.

[9] Kamiokande, Y. Fukuda et al., Phys. Lett. B335 (1994) 237.

[10] IMB, R. Becker-Szendy et al., Phys. Rev. D46 (1992) 3720.

[11] Super-Kamiokande, Y. Fukuda et al., Phys. Rev. Lett. 81 (1998) 1562, hep-ex/9807003.

[12] Soudan 2, M. Sanchez et al., Phys. Rev. D68 (2003) 113004, hep-ex/0307069.

[13] MACRO, M. Ambrosio et al., Phys. Lett. B566 (2003) 35, hep-ex/0304037.

[14] MINOS, P. Adamson et al., Phys. Rev. D86 (2012) 052007, arXiv:1208.2915.

[15] K2K, M.H. Ahn et al., Phys. Rev. D74 (2006) 072003, hep-ex/0606032.

[16] MINOS, P. Adamson et al., Phys. Rev. Lett. 108 (2012) 191801, arXiv:1202.2772.

[17] Super-Kamiokande, K. Abe et al., Phys.Rev.Lett. 110 (2013) 181802, arXiv:1206.0328.

[18] OPERA, N. Agafonova et al., Phys.Rev. D89 (2014) 051102, arXiv:1401.2079.

[19] T2K, K. Abe et al., Phys. Rev. Lett. 107 (2011) 041801, arXiv:1106.2822.

[20] MINOS, P. Adamson et al., Phys. Rev. Lett. 107 (2011) 181802, arXiv:1108.0015.

[21] T2K, K. Abe et al., Phys.Rev.Lett. 112 (2014) 061802, arXiv:1311.4750.

[22] Daya Bay, F.P. An et al., Phys. Rev. Lett. 108 (2012) 171803, arXiv:1203.1669.

[23] RENO, S.B. Kim et al., Phys. Rev. Lett. 108 (2012) 191802, arXiv:1204.0626.

[24] Double Chooz, Y. Abe et al., Phys.Lett. B723 (2013) 66, arXiv:1301.2948.

[25] Daya Bay, F. An et al., Phys.Rev.Lett. 112 (2014) 061801, arXiv:1310.6732.

[26] M. Mezzetto and T. Schwetz, J. Phys. G37 (2010) 103001, arXiv:1003.5800.

[27] F. Capozzi et al., Phys.Rev. D89 (2014) 093018, arXiv:1312.2878.

[28] NuFIT, http://www.nu-fit.org/.

[29] M. Gonzalez-Garcia et al., JHEP 12 (2012) 123, arXiv:1209.3023.

[30] G. Mention et al., Phys. Rev. D83 (2011) 073006, arXiv:1101.2755.

[31] T.A. Mueller et al., Phys. Rev. C83 (2011) 054615, arXiv:1101.2663.

[32] P. Huber, Phys. Rev. C84 (2011) 024617, arXiv:1106.0687.

[33] SAGE, J.N. Abdurashitov et al., Phys. Rev. C73 (2006) 045805, nucl-ex/0512041.

[34] M. Laveder, Nucl. Phys. Proc. Suppl. 168 (2007) 344.

[35] C. Giunti and M. Laveder, Mod. Phys. Lett. A22 (2007) 2499, hep-ph/0610352.

[36] C. Giunti and M. Laveder, Phys. Rev. C83 (2011) 065504, arXiv:1006.3244.

[37] C. Giunti et al., Phys. Rev. D86 (2012) 113014, arXiv:1210.5715.

[38] F. Kaether et al., Phys. Lett. B685 (2010) 47, arXiv:1001.2731.

[39] SAGE, J.N. Abdurashitov et al., Phys. Rev. C80 (2009) 015807, arXiv:0901.2200.

[40] LSND, C. Athanassopoulos et al., Phys. Rev. Lett. 75 (1995) 2650, nucl-ex/9504002.

[41] LSND, A. Aguilar et al., Phys. Rev. D64 (2001) 112007, hep-ex/0104049. 
[42] N. Okada and O. Yasuda, Int. J. Mod. Phys. A12 (1997) 3669, hep-ph/9606411.

[43] S.M. Bilenky, C. Giunti and W. Grimus, Eur. Phys. J. C1 (1998) 247, hep-ph/9607372.

[44] S.M. Bilenky et al., Phys. Rev. D60 (1999) 073007, hep-ph/9903454.

[45] M. Maltoni et al., New J. Phys. 6 (2004) 122, hep-ph/0405172.

[46] M. Sorel, J. Conrad and M. Shaevitz, Phys. Rev. D70 (2004) 073004, hep-ph/0305255.

[47] G. Karagiorgi et al., Phys. Rev. D75 (2007) 013011, hep-ph/0609177.

[48] M. Maltoni and T. Schwetz, Phys. Rev. D76 (2007) 093005, arXiv:0705.0107.

[49] G. Karagiorgi et al., Phys. Rev. D80 (2009) 073001, arXiv:0906.1997.

[50] A.E. Nelson, Phys. Rev. D84 (2011) 053001, arXiv:1010.3970.

[51] J. Fan and P. Langacker, JHEP 04 (2012) 083, arXiv:1201.6662.

[52] E. Kuflik, S.D. McDermott and K.M. Zurek, Phys. Rev. D86 (2012) 033015, arXiv:1205.1791.

[53] J. Huang and A.E. Nelson, Phys.Rev. D88 (2013) 033016, arXiv:1306.6079.

[54] B. Pontecorvo, Sov. Phys. JETP 26 (1968) 984.

[55] R.R. Volkas, Prog. Part. Nucl. Phys. 48 (2002) 161, hep-ph/0111326.

[56] R.N. Mohapatra and A.Y. Smirnov, Ann. Rev. Nucl. Part. Sci. 56 (2006) 569, hep-ph/0603118.

[57] A. Diaferio and G.W. Angus, arXiv:1206.6231.

[58] J. Lesgourgues et al., Neutrino Cosmology (Cambridge University Press, 2013).

[59] S. Riemer-Sorensen, D. Parkinson and T.M. Davis, Publ.Astron.Soc.Austral. 30 (2013) e029, arXiv:1301.7102.

[60] M. Archidiacono et al., Adv.High Energy Phys. 2013 (2013) 191047, arXiv:1307.0637.

[61] J. Lesgourgues and S. Pastor, New J.Phys. 16 (2014) 065002, arXiv:1404.1740.

[62] M.C. Gonzalez-Garcia and M. Maltoni, Phys. Rept. 460 (2008) 1, arXiv:0704.1800.

[63] J. Conrad et al., Adv.High Energy Phys. 2013 (2013) 163897, arXiv:1207.4765.

[64] J. Kopp et al., JHEP 1305 (2013) 050, arXiv:1303.3011.

[65] C. Giunti et al., Phys.Rev. D88 (2013) 073008, arXiv:1308.5288.

[66] MiniBooNE, A.A. Aguilar-Arevalo et al., Phys. Rev. Lett. 102 (2009) 101802, arXiv:0812.2243.

[67] MiniBooNE, A.A. Aguilar-Arevalo et al., Phys. Rev. Lett. 105 (2010) 181801, arXiv:1007.1150.

[68] MiniBooNE, A. Aguilar-Arevalo et al., Phys.Rev.Lett. 110 (2013) 161801, arXiv:1303.2588.

[69] C. Giunti and M. Laveder, Phys.Rev. D84 (2011) 093006, arXiv:1109.4033.

[70] C. Giunti and M. Laveder, Phys. Lett. B706 (2011) 200, arXiv:1111.1069.

[71] M. Martini, M. Ericson and G. Chanfray, Phys. Rev. D85 (2012) 093012, arXiv:1202.4745.

[72] M. Martini, M. Ericson and G. Chanfray, Phys. Rev. D87 (2013) 013009, arXiv:1211.1523.

[73] BNL-E776, L. Borodovsky et al., Phys. Rev. Lett. 68 (1992) 274.

[74] KARMEN, B. Armbruster et al., Phys. Rev. D65 (2002) 112001, hep-ex/0203021. 
[75] NOMAD, P. Astier et al., Phys. Lett. B570 (2003) 19, hep-ex/0306037.

[76] ICARUS, M. Antonello et al., Eur.Phys.J. C73 (2013) 2599, arXiv:1307.4699.

[77] OPERA, N. Agafonova et al., JHEP 1307 (2013) 004, arXiv:1303.3953.

[78] M.A. Acero, C. Giunti and M. Laveder, Phys. Rev. D78 (2008) 073009, arXiv:0711.4222.

[79] CDHSW, F. Dydak et al., Phys. Lett. B134 (1984) 281.

[80] A. Esmaili, F. Halzen and O.L.G. Peres, JCAP 1211 (2012) 041, arXiv:1206.6903.

[81] A. Esmaili and A.Y. Smirnov, JHEP 1312 (2013) 014, arXiv:1307.6824.

[82] MINOS, P. Adamson et al., Phys. Rev. Lett. 107 (2011) 011802, arXiv:1104.3922.

[83] SciBooNE-MiniBooNE, K.B.M. Mahn et al., Phys. Rev. D85 (2012) 032007, arXiv:1106.5685.

[84] SciBooNE-MiniBooNE, G. Cheng et al., Phys. Rev. D86 (2012) 052009, arXiv:1208.0322.

[85] M. Maltoni and T. Schwetz, Phys. Rev. D68 (2003) 033020, hep-ph/0304176.

[86] M. Archidiacono et al., Phys.Rev. D87 (2013) 125034, arXiv:1302.6720.

[87] J. Kopp, M. Maltoni and T. Schwetz, Phys. Rev. Lett. 107 (2011) 091801, arXiv:1103.4570.

[88] C. Giunti and M. Laveder, Phys.Rev. D84 (2011) 073008, arXiv:1107.1452.

[89] M. Archidiacono et al., Phys. Rev. D86 (2012) 065028, arXiv:1207.6515.

[90] K.N. Abazajian et al., arXiv:1204.5379.

[91] C. Rubbia et al., arXiv:1304.2047.

[92] OscSNS, M. Elnimr et al., arXiv:1307.7097.

[93] J.P. Delahaye et al., arXiv:1308.0494.

[94] LArTPC, B. Fleming, O. Palamara and . D.Schmitz, arXiv:1309.7987.

[95] M. Antonello et al., arXiv:1312.7252.

[96] nuSTORM, D. Adey et al., Phys.Rev.D (2014), arXiv:1402.5250.

[97] C. Rubbia, arXiv:1408.6431. 